\title{
New Data Analysis Tools for X-ray Photoelectron Spectroscopy (XPS) and Spectroscopic Ellipsometry (SE): Uniqueness Plots and Width Functions in XPS, and Distance, Principal Component, and Cluster Analyses in SE
}

\author{
Matthew R. Linford, ${ }^{1}$ Bhupinder Singh, ${ }^{1}$ Daniel Velázquez, ${ }^{2}$ Jeff Terry, ${ }^{2}$ Jacob D. Bagley, ${ }^{1}$ Dennis H. \\ Tolley, ${ }^{3}$ Anubhav Diwan, ${ }^{1}$ Varun Jain, ${ }^{1}$ Alberto Herrera-Gomez ${ }^{4}$ \\ ${ }^{1}$ Departments of Chemistry and Biochemistry and ${ }^{3}$ Statistics, Brigham Young University, UT, USA \\ ${ }^{2}$ Department of Physics, Illinois Institute of Technology, Chicago, IL, USA \\ ${ }^{4}$ CINVESTAV-Unidad Queretaro, Queretaro, Mexico
}

Here we discuss a series of data analysis tools for X-ray photoelectron spectroscopy (XPS) and spectroscopic ellipsometry (SE). For XPS, these include uniqueness plots [1], and the equivalent [2] and autocorrelation [3] widths. For SE, they include distance, principal component, and cluster analyses [4].

Uniqueness plots are widely used in the SE community for identifying correlation between fit parameters [5]. They are easily interpreted. However, they appear not to have been employed for XPS data analysis. And certainly better tools are needed to identify inappropriate peak fits to XPS narrow scans because (i) XPS is now receiving in excess of 10,000 mentions in the literature each year [6], and (ii) with the proliferation of the technique, the number of untrained users that are collecting and fitting data has significantly increased. In a number of reported peak fits, too many fit parameters have been introduced into the data modeling, which has reduced or eliminated the statistical meaning of these parameters.

Uniqueness plots show the error of a fit as a function of one of the variables in that fit, where the values of a specified variable are systematically fixed to quantities about its optimal value. If the same, low error can be obtained for all the values of the variable in question, a horizontal line is obtained, which signals fit parameter correlation. Here, the same error is obtained because other variables in the fit are able to compensate for the systematic change to the variable in question. In contrast, if the error in the fit rises as the variable in question is systematically changed about its optimal value, the fit has uniqueness, i.e., the other variables in the fit are unable to compensate for changes in the variable in question. Uniqueness plots that indicate the absence of fit parameter correlation are often parabolic in shape. We have applied uniqueness plots to the peak fitting of XPS C 1s narrow scans of ozone-treated carbon nanotube (CNT) forests that were obtained as part of a study on CNT-templated thin layer chromatography plates [7], and Si 2p narrow scans of oxidized silicon. In both cases, uniqueness plots showed that unconstrained fits had poor uniqueness, while more reasonably constrained fits had better uniqueness. These results indicate that uniqueness plots may be a valuable tool for identifying inappropriate peak fits in XPS.

SE determines two parameters, $\Psi$ and $\Delta$, as a function of wavelength, where tan $\Psi$ refers to the ratio of the amplitudes of the $\mathrm{p}$ - and s-polarized light impinging on a surface, and $\Delta$ refers to the phase difference between these waves. It is fairly common practice within the SE community to evaluate material stability, or changes in a material, by plotting these two parameters at a few wavelengths as a function of time or some other variable of interest. In general, if $\Psi$ and $\Delta$ in these plots remain constant, or essentially constant, the material is deemed to be stable. We recently questioned this conventional approach, which only relies on a very small subset of the SE data that are collected. Accordingly, we 
applied distance, principal component, and cluster analyses to a set of data we had used to 'confirm' the stability of a thin, sputtered film of bismuth, tellurium, and selenium, which had been developed as a possible data storage layer [8]. Interestingly, when the entire data sets were considered, or a much larger fraction of them, a substantial change was observed in the data that was otherwise difficult to observe. This approach was also applied to a second set of data that similarly showed no change by the conventional approach but a noticeable change when the more advanced statistical analysis was performed. Both range selection of the data and a weighted distance formula were considered.

Time permitting, the use of the equivalent and autocorrelation widths in XPS narrow scan analysis will also be discussed. These are unbiased tools that signal chemical changes in materials.

\section{References:}

[1] B Singh, A Diwan, V Jain, A Herrera-Gomez, J Terry, MR Linford, Submitted to Applied Surface Science.

[2] B Singh, D Velázquez, J Terry and MR Linford, J. Electron Spectrosc. 197 (2014), 56-63.

[3] B Singh, D Velázquez, J Terry and MR Linford, J. Electron Spectrosc. 197 (2014), 112-117.

[4] JD Bagley, DH Tolley, MR Linford, Surface and Interface Analysis (2016), DOI: 10.1002/sia.5938.

[5] JN Hilfiker, N Singh, T Tiwald, D Convey, SM Smith, JH Baker and HG Tompkins, Thin Solid Films 516 (2008), 7979-7989.

[6] CJ Powell, J. Vac. Sci. \& Technol. A 21 (2003), S42 - S53.

[7] DS Jensen, SS Kanyal, V Gupta, MA Vail, AE Dadson, M Engelhard, R Vanfleet, RC Davis, MR Linford, J. Chrom. A. 1257 (2012), 195 - 203.

[8] H Wang, N Madaan, J Bagley, A Diwan, Y Liu, RC Davis, BM Lunt, SJ Smith, MR Linford, Thin Solid Films 569 (2014), 124 - 130. 